\title{
Special Session 3 \\ The Virtual Observatory in action: new science, new technology, and next generation facilities
}

\author{
Nicholas A. Walton ${ }^{1}$, Andrew Lawrence ${ }^{2}$, and Roy Williams ${ }^{3}$ (eds.) \\ ${ }^{1}$ Institute of Astronomy, University of Cambridge, Madingley Road, Cambridge CB3 0HA, UK \\ email: naw@ast.cam.ac.uk \\ ${ }^{2}$ University of Edinburgh, Royal Observatory, Blackford Hill, Edinburgh EH9 3HJ, UK \\ email: al@roe.ac.uk \\ ${ }^{3}$ Center for Advanced Computer Research, California Institute of Technology, \\ MC 158-79, 1200 E. California Boulevard, Pasadena, CA 91125, USA \\ email: roy@cacr.caltech.edu
}

\section{Preface}

The vision of the Virtual Observatory (VO) is to make access to astronomical databases as seamless and transparent as browsing the World Wide Web is today. It will federate the data flows from current and future facilities and large scale surveys, and the computational resources and new tools necessary to fully exploit them. This requires both technological developments and an international commitment to standardisation and working culture. Increasingly, it will alter the way that astronomers do science, and the way that future facilities and projects plan for their data management, and the scientific exploitation of their data. It will make an impact on a wide variety of astronomical topics, but especially those using very large databases, and those needing a multiwavelength approach, or more generally the use of multiple archives.

To date, there are fifteen VO projects worldwide, who co-ordinate their efforts through an International Virtual Observatory Alliance (IVOA). This body evolves and agrees technical standards as well as sharing best practice and software. The various VO projects have laid the foundations for the VO: international standards, fundamental infrastructure, early demonstrations, and the first published science papers using VO tools. The $\mathrm{VO}$ is now becoming an operational reality. The next stage involves $(i)$ deployment of the new infrastructure at data centres; $(i i)$ science results enabled via the use of VO services; (iii) making links to existing and planned facilities; and (iv) much more ambitious data mining analysis services.

Following endorsement and sponsoring by IAU Divisions and Commissions and subsequent approval by the IAU Executive Committee, this IAU Special Session on 'The Virtual Observatory in Action' allowed for the latest developments in the area of the Virtual Observatory to be presented. Talks and posters covered an exciting range covering new science results, plans for major facilities, and discussion of technical advances in data mining and the VO.

The presentations given here allow for a brief summary of the range of topics covered. We have also made the slides for all the oral presentations given at this Special Session available at the SPS 3 conference website:

<http://www.ivoa.net/pub/VOScienceIAUPrague/programme/index.html>. 
The smooth running of the VO Special Session, organised as one of the constituent sessions of the IAU XXVI General Assembly in Prague, August 2006, greatly benefited from the excellent local organisation. We greatly acknowledge the help of Prof. Meszaros and the many helpful local students and staff, along with the session chairs, in ensuring the smooth running of this Special Session.

\section{Scientific Organizing Committee}

Giuseppina Fabbiano (USA), Françoise Genova (France), Robert J. Hanisch (USA), Ajit K. Kembhavi (India), Andrew Lawrence (UK, chair), Oleg Yu. Malkov (Russia), Atilla Meszaros (Czech Republic), Raymond P. Norris (Australia), Masatoshi Ohishi (Japan), Peter Quinn (Germany), Isabelle F. Scholl (France), Enrique Solano (Spain), Alexander Szalay (USA), Nicholas A. Walton (UK), Roy Williams (USA), and Yongheng Zhao (China).

Andrew Lawrence, chair SOC,

Nicholas Walton 8 Roy Williams, editors,

Edinburgh, Cambridge, Pasadena, November 30, 2006 